\title{
Impacto social del Covid-19 en un contexto de informalidad
}

\section{Social impact of Covid-19 in a context of informality}

\author{
AVENDAÑO-CASTRO, William R. ${ }^{1}$ \\ HERNÁNDEZ-SUÁREZ, César A. ${ }^{2}$ \\ PRADA-NÚÑEZ, Raúl ${ }^{3}$
}

\begin{abstract}
Se determinan los impactos socio-económicos y educativos del Covid-19 en un grupo de individuos pertenecientes al sector informal. Corresponde a un estudio cuantitativo de nivel descriptivo y de tipo no experimental, transeccional. La muestra estuvo conformada por 199 sujetos. Se empleó un cuestionario como instrumento de recolección de datos. Los resultados muestran algunos problemas, por ejemplo, debilidades en el aprendizaje real desde casa. Se concluye que las desigualdades sociales, económicas y educativas en este contexto crean barreras y limitaciones.

key words: coronavirus, covid-19, impacto socioeconómico, impacto educativo

\section{Resumen}

The socio-economic and educational impacts of Covid-19 are determined in a group of individuals belonging to the informal sector. It corresponds to a quantitative study of a descriptive level and of a non-experimental, transectional type. The sample consisted of 199 subjects. A questionnaire was used as a data collection instrument. The results show some problems, for example, weaknesses in real learning from home. It is concluded that social, economic and educational inequalities in this context create barriers and limitations.

Palabras clave: coronavirus, covid-19, socioeconomic impact, educational impact
\end{abstract}

\section{Introducción}

El coronavirus ha afectado la vida del ser humano en diversos ámbitos y de diferentes maneras. Por ejemplo, ha tenido un enorme impacto negativo en el ámbito económico y social (Gambau, 2020). En el escenario educativo, el Covid-19 ha afectado a millones de niños y jóvenes. Aproximadamente en 107 países se implementó como estrategia preventiva el cierre de las escuelas. Como resultado de la crisis provocada por la emergencia sanitaria, se ha adoptado un modelo de educación a distancia y remoto para continuar con los procesos de formación y los planes de estudio. Esto ha traido limitaciones, por las condiciones y situaciones particulares tanto de estudiantes como docentes: "la educación a distancia es una quimera, una alternativa para los que tienen equipos de una cierta calidad con acceso a internet en casa, [...] pero con estudiantes que no cuentan con las

\footnotetext{
${ }^{1}$ Docente Investigador. Facultad de Ciencias Empresariales. Universidad Francisco de Paula Santander, Cúcuta, Colombia. williamavendano@ufps.edu.co 2 Docente Investigador. Facultad de Educación, Artes y Humanidades. Universidad Francisco de Paula Santander, Cúcuta, Colombia. cesaraugusto@ufps.edu.co

${ }^{3}$ Docente Investigador. Facultad de Educación, Artes y Humanidades. Universidad Francisco de Paula Santander, Cúcuta, Colombia. raulprada@ufps.edu.co
} 
condiciones [...] para poder beneficiarse de esta opción" (Murillo \& Duk, 2020, p.12). El Covid-19 ha configurado brechas educativas que se han hecho más relevantes y evidentes en medio de la crisis.

Por otra parte, en el contexto de informalidad, la pandemia trae a la par impactos significativos en términos de desempleo y subempleo, especialmente en los trabajadores informales. De la misma manera, la dinámica de la pandemia trae consigo consecuencias negativas en todos los sectores de la fuerza laboral, incluido el educativo, así como una crisis social y económica. Al mismo tiempo, "la informalidad, además de la inseguridad e inestabilidad laboral, está asociada con bajos ingresos y, por lo tanto, con una baja capacidad de ahorro" (Álvarez et al., 2020, p.10). Por otro lado, la Comisión Económica para América Latina y el Caribe (CEPAL) y la Oficina para el Cono Sur de América Latina de la Organización Internacional del Trabajo (OIT), "prevé que la actividad económica de la región se contraiga el 5,3\% en 2020" (p. 5), como resultado de una inevitable extensión de la pandemia y el necesario distanciamiento social. Esta dinámica es alarmante en el contexto de un mercado laboral con una alta incidencia de empleo en la informalidad y empleo formal frágiles.

De acuerdo con la OIT (2020) se estima un aumento entre 5,3 millones y 24,7 millones de desempleo debido a la crisis actual. Por otra parte, la CEPAL (2020) señala que el Covid-19 "afectará el número de empleos, la calidad del trabajo (reducción de salarios y menor acceso a la protección social) y a los grupos más vulnerables, como los trabajadores en el sector informal"(p. 5). Sin duda, la crisis sanitaria impactará la cotidianidad de las personas en diferentes niveles, incluso, lo educativo, dadas las implicaciones sociales y emocionales que causan el confinamiento y la crisis en los hogares. Los niños y jóvenes, perceptivos de estas realidades, tienen limitaciones para trabajar desde casa en las tareas académicas designadas.

En este contexto, los trabajadores informales, ya sean asalariados o independientes, se hallan entre los grupos vulnerables con mayor riesgo de perder sus empleos e ingresos. Incluso, en opinión de Álvarez et al. (2020), entre este "grupo de trabajadores independientes, se debe hacer una distinción entre profesionales independientes, [...] Solo el $14 \%$ de los trabajadores independientes han estudiado en instituciones de educación superior, mientras que alrededor del $56 \%$ logra una educación secundaria". Igualmente argumentan que los ingresos se encuentran condicionados al factor educativo: aquellos que terminan la escuela secundaria, el ingreso promedio en el 2019 fue de 634,589 pesos mensual. Esto representa, aproximadamente, un ingreso laboral equivalente a un $14 \%$ menos que el salario mínimo. En contraste, quienes tenían una educación superior tenían un ingreso laboral mensual promedio de 1,164,886 pesos en 2019 (40\% por encima del salario mínimo legal).

Por definición, no disfrutan de contratos de trabajo seguros y, por ende, no gozan de los beneficios de los trabajadores y protecciones sociales. Esto hace aún más sensibles a los shocks económicos, sociales y educativos provocados por la pandemia Covid-19. Puesto que se enmarcan en la participación de los procesos de flexibilización curricular del plan de estudio de sus hijos. La educación desde la casa podría considerarse bastante positiva, ya que es probable que sea efectiva. Pero, por lo general, esta modalidad se ve como un complemento de los aportes de la escuela. Aunque "parar abruptamente las clases, a la larga podría arrojar un resultado negativo y perjudicial por el desgaste, tanto para el alumno, los padres, los docentes"(Britez, 2020, p.13). Sin embargo, en opinión de Razeto (2016) "las iniciativas y programas para fortalecer el rol educativo de la familia, han sido insuficientes. [...] el involucramiento de la familia es uno de los predictores más potentes del éxito escolar, <pero> han sido pocos, débiles e inconsistentes".

A pesar de que diversas naciones han acogido con importancia situar a los habitantes informales y la fuerza laboral en el mapa, y en el presupuesto, "El impacto de cierre de escuelas y otras restricciones sociales ha ocasionado en los grupos más desventajados, está suscitando gran interés y preocupación a nivel global". (Murillo \& Duk, 2020, p.13). Entonces, la transición hacia la educación a distancia de emergencia desde casa se ha unido a otros impactos no menos importantes entre los distintos actores, si bien posiblemente menos perceptibles y documentados. Estos otros impactos son susceptibles de darse en ámbitos como el 
socioeconómico, el laboral, e, innegablemente, relativo al trabajo y del sistema en su conjunto debido a una brecha digital que enfrenta también notables desafíos territoriales.

Según datos del Departamento Administrativo Nacional de Estadística DANE (2018) en el Boletín Técnico de Indicadores Básicos de Tenencia y uso de Tecnologías de la Información y Comunicación (TIC), en hogares y personas de 5 y más años de edad, en el 78,1\% de los hogares colombianos existe un teléfono celular inteligente, no obstante, el resto posee teléfono celular convencional. Mientras que la proporción porcentual de hogares a nivel nacional que poseen computador de escritorio es de $28,8 \%$, portátil $(20,6 \%)$ o tableta $(10,9 \%)$. Entretanto, que la proporción de hogares que poseen conexión a Internet según tipo de conexión a nivel nacional es Hogares con Internet, 52,7\%; Hogares con Internet - Fijo, 40,5\%, y Hogares con Internet - Móvil, 29,2\%.

De este modo, las implicaciones socioeconómicas y educativas originadas por el Covid-19, han originado respuestas políticas inmediatas para apoyar el empleo y evitar los niveles de desempleo e informalidad. Asimismo, se han fijado decretos, resoluciones y circulares que establecen medidas de protección al empleo y educativas con ocasión de la fase de contención del Covid-19 y de la declaración de estado de emergencia sanitaria, en lo que destacan "el Estado de emergencia económica, social y ecológica" (Decreto 417, 2020; Decreto 637, 2020) y "que el sistema educativo oficial con ocasión de las medidas adoptadas para prevenir la propagación del Covid-19, deberá ser atendido a través de estrategias pedagógicas flexibles coordinadas con las 96 Secretarías de Educación certificadas"(Decreto 470, 2020). Por otra parte, García \& Kairuz (2020) exponen que en Colombia, aproximadamente de 8 millones de estudiantes que están matriculados en los colegios oficiales, 5 millones y medio son beneficiarios del Programa de Alimentación Escolar (PAE). Estos autores señalan que según el ejecutivo se mantendrían los componentes nutricional y de educación, el pago por los niños beneficiarios del programa Familias en Acción, aunque es posible que en la focalización de estas medidas se deje por fuera una parte importante de la población vulnerable.

Así mismo, se han expedido la Circular 021 (2020), la cual adopta medidas de protección al empleo con ocasión de la fase de contención de Covid-19 y de la declaración de emergencia sanitaria; el Decreto 488 (2020), por el cual se dictan medidas de orden laboral, dentro del Estado de Emergencia Económica, Social y Ecológica porque "el desempleo en Colombia genera una perturbación grave y extraordinaria en el orden económico y social, así como en su Producto Interno Bruto" (Decreto 639, 2020). Sin embargo, más allá de los riesgos de contagio, el impacto de Covid-19 en la economía colombiana pudiera venir en tres oleadas: (i) disminución de la tasa de ocupados; (ii) un aumento en el desempleo asociada con los cierres de comercios y despidos de trabajadores y (iii) un shock de informalidad que afecta el comercio interno colombiano. Por ello, el Ministerio de Trabajo (2020), a través del Decreto 801 (2020) "crea el auxilio económico a la población cesante" como medida de mejorar el bienestar de los extrabajadores y asegurar la participación activa de las personas en los diferentes ámbitos de la sociedad, en especial, el educativo.

Con respecto al ámbito educativo y de la informalidad, en el aspecto económico la Presidencia de la República a través del Decreto 417 (2020), señala que "el 42,4\% de los trabajadores en Colombia trabajan por cuenta propia y $56,4 \%$ no son asalariados, los ingresos de este tipo de trabajadores y sus dependientes dependen de su trabajo diario y esta actividad se ha visto restringida por las medidas de la pandemia". Adicionalmente, argumenta que los hogares son vulnerables al no contar con mecanismos para reemplazar los ingresos que dejarán de percibir por causa de las medidas sanitarias. Por otro lado, en el aspecto educativo en dicho país se cuenta "en educación preescolar, básica y media a 6.928 .742 niños, niñas y adolescentes" (Decreto 470, 2020).

Por otro lado, el DANE (2020) muestra que la población ocupada formal e informal para el mes de mayo del presente año, tuvo una disminución con respecto al año 2019. De allí que "la población ocupada informal en las 13 ciudades y áreas metropolitanas fue 3.870 miles de personas" en mayo de 2020, con una variación negativa de $(-1,181)$ con respecto a los 5.051 miles de personas de mayo de 2019. De igual modo, sucedió "para el total 
de las 23 ciudades y áreas metropolitanas fue 4.300 miles de personas" (mayo 2019), con una variación negativa de ocupados informales de $(-1,440)$ con respecto a los 5.740 miles de personas ocupadas informales en mayo de 2019. De igual manera, argumenta que para la misma fecha (mayo de 2020), la simetría de hombres ocupados informales en las mismas ciudades y áreas metropolitanas fue de 45,9\%, para las mujeres ocupadas informales. Por tanto, la situación laboral, en Colombia, representan los desajustes en el mercado laboral entre el desempleo y la informalidad (García, 2008).

De allí que el contexto de la informalidad se verá afectado socialmente, además de que esta población muchas veces con pocos estudios académicos, ahora deben considerar la situación disruptiva de cierre de escuelas durante largos períodos en el sistema educativo, y que pudiera traer impactos negativos en el aprendizaje de sus hijos, especialmente para los grupos vulnerables. Las altas tasas de informalidad ocurren principalmente en el comercio minorista, la construcción y las actividades de servicio doméstico (Álvarez et al., 2020). Asimismo, estos autores añaden que estas son actividades que se vieron directamente afectadas por el bloqueo y que, con la excepción de la construcción, seguramente permanecerán inactivas hasta el final de la crisis actual.

De lo anteriormente expuesto, la ciudad fronteriza de Cúcuta no escapa a esta realidad, incluso, en opinión de Peralta \& Bayona (2016) el crecimiento socioeconómico en Cúcuta tiene como barrera la informalidad empresarial. Aunque, la "informalidad no depende sólo de empresas sino también de las personas ya que al cumplir su ciclo de vida laboral buscan la manera de incrementar sus ingresos y de ampliar su condición laboral por medio de la creación de negocios" (Montañez, 2016, p. 61). Además, este autor argumenta que dichas empresas o negocios, así como trabajadores, no están oficialmente en el marco de regulación jurídica en Colombia que determina la protección del trabajador informal y de esta manera garantizar al trabajador informal el acceso a los mecanismos propios para la administración de justica en base a los derechos laborales.

Al respecto, la Cámara de Comercio de Cúcuta (2019a) menciona que Cúcuta en "la informalidad, el promedio oscila entre el $68 \%$ y $71 \%$, siendo la ciudad con mayor población informal del país". Asimismo, según la Cámara de Comercio de Cúcuta (2019b), en el Municipio hay 60 establecimientos educativos oficiales y 210 no oficiales, de los cuales hay en zonas urbanas 153.798 estudiantes y en zona rurales 7.022 estudiantes. Mientras que las estadísticas generales de la Subdirección de Desarrollo Sectorial de Educación Superior a través del Sistema Nacional de Información de la Educación Superior (SNIES, 2018) menciona que en la ciudad de Cúcuta, existían matriculados 42.108 estudiantes universitarios para el año 2018.

La informalidad está especialmente extendida en diversos sectores y particularmente difícil es la situación de estos trabajadores que representan casi la mitad de la fuerza laboral total, pero siguen siendo en gran medida invisibles, incluso, un nutrido porcentaje se encuentra entre los trabajadores más pobres, y a menudo están empleados de manera estacional o temporal. El impacto económico en las personas exacerba las vulnerabilidades existentes, incluida la pobreza, el desempleo, la exclusión social y educativa desde casa a través de la tecnología y modalidad a distancia o remota. De allí que las epidemias y las crisis económicas tienen un impacto desproporcionado en los segmentos más vulnerables de la población cucuteña, puesto que, estos trabajadores apenas ganan suficiente salario para alimentarse a sí mismos y a sus familiares, lo que puede provocar una mayor desigualdad social en acceso a educación, seguridad alimentaria, así como, en la percepción de la situación de empleo y desempleo.

La importancia de esta investigación radica en la percepción que tienen las personas del contexto de informalidad en la Ciudad de Cúcuta con respecto a los impactos socio-económicos y educativos causados por la pandemia y el estado de emergencia sanitaria. Al mismo tiempo, los resultados generados pueden contribuir a establecer un aporte a la literatura científica en el ámbito de la informalidad y la educación. El objetivo fue determinar la percepción en los individuos del contexto de informalidad, sobre los impactos socio-económicos y educativos de Covid-19 en la Ciudad de Cúcuta, Norte de Santander, Colombia. 


\section{Metodología}

La investigación es de tipo descriptivo, con un diseño no experimental, transeccional y de campo. De acuerdo con Hernández et al. (2014) los estudios descriptivos "buscan especificar las propiedades, las características y los perfiles de personas, grupos, comunidades, procesos, objetos o cualquier otro fenómeno que se someta a un análisis"(p. 92). La población estuvo constituida 409 ciudadanos que se encuentran económicamente en la informalidad en la Ciudad de Cúcuta, Colombia. Los datos poblacionales se lograron a través de una encuesta online para personas que estén laboralmente ocupados o no, jubilados o estudiando.

Por tanto, la población se clasifica como finita (Arias, 2012) y "esta queda delimitada por el problema y por los objetivos del estudio"(p. 82).Para obtener el tamaño de la muestra en poblaciones finitas, que es el caso, se siguió el procedimiento referenciado por Bernal (2010), con un valor para $p=0.50$, un margen de error del $5 \%$ y un nivel de confianza del 95\%. El resultado obtenido fue de 199 sujetos.

Se utilizó como técnica de recolección de datos la encuesta. Según Arias (2012), un instrumento de recolección de datos "es cualquier recurso, dispositivo o formato (en papel o digital), que se utiliza para obtener, registrar o almacenar información" (p. 67). Para la recopilación de la información de la investigación, se elaboró un cuestionario. El instrumento fue autoadministrado de manera online estructurado con 24 preguntas con varias opciones de respuesta.

En cuanto al procedimiento de recolección de la información, los cuestionarios se aplicaron de manera online, durante el mes de abril y mayo de 2020. Respondieron a los cuestionarios un total de 921 habitantes de la ciudad de Cúcuta, de los cuales 409 pertenecen al sector económico de informalidad (población objeto de estudio), lo que representó un $44,40 \%$ de encuestas válidas y la cantidad necesaria para cubrir el tamaño de la muestra obtenida. Los datos recolectados fueron organizados y tabulados en hojas de cálculo del programa Excel y a través de la "estadística se procesan los datos obtenidos para describir, organizar, analizar e interpretar en forma apropiada los resultados" (Tamayo \& Tamayo, 2007, p.143). Se efectúo una exploración descriptiva en el cuestionario en el que se incluyeron estadísticos como la frecuencia para el análisis e interpretación de los datos.

\section{Resultados y discusión}

A continuación se hace una descripción de los análisis de los resultados obtenidos y el procesamiento de los datos sobre el cuestionario "Impacto socioeconómico educativo de Covid-19", en contexto de informalidad, aplicado en la Ciudad de Cúcuta. El 52,8\% de los participantes correspondió a mujeres, y 47,2\%, a hombres. En lo relativo a la edad, el porcentaje de participantes según rango de edad fue: 12 a 20 años (23,6\%); 21 a 30 años (31,7\%); 31 a 40 años (16,1\%); 41 a 50 años (18,6\%) y 51 años o más (10,1\%). De igual manera, se determinó que $59,3 \%$ de los encuestados tiene ubicada su residencia en Cúcuta, mientras que el resto tiene ubicado su hogar en Los Patios (24,1\%), Villa del Rosario $(13,6 \%)$ y otros lugares aledaños a la ciudad de Cúcuta $(3,0 \%)$.

Se logró verificar que 50,8\% de los trabajadores de informalidad encuestados tiene como estado civil 'divorciado', mientras que un $33,2 \%$ expresó que se encuentra casado/concubinato, y el resto, soltero (16,1\%). En lo referente, a cuántas personas dependen económicamente de dichos trabajadores en el hogar, se pudo constatar que 23,1\% no posee carga familiar, mientras que $16,6 \%$ señaló que depende de ellos una sola persona. El resto expresó que en su hogar dependen de sus trabajos dos personas $(21,1 \%)$; tres personas $(17,6 \%)$; cuatro personas $(12,1 \%)$; cinco personas $(5,5 \%)$, seis personas $(3,0 \%)$; siete personas $(0,5 \%)$ y ocho personas $(0,5 \%)$.

En cuanto al nivel de estudio completado se obtuvo que 50,8\% ha terminado la educación media, mientras que 26,6\% señaló que tiene un nivel de educación técnico laboral. Un 14,1\% manifestó ser profesional universitario, y 2,0\% expresó que posee una especialización. El resto, 5,0\% terminó la educación primaria y 1,5\% no posee 
ningún estudio académico completado. Lo cual concuerda con Álvarez et al. (2020) quienes señalan que 14\%de los trabajadores de informalidad ha estudiado en instituciones de educación superior, lo que podría tener relación con el estado de vulnerabilidad debido a bajos ingresos con respecto a la escolaridad y autoempleo de dichos trabajadores, y que podría incidir negativamente al afrontar una educación desde la casa por los bienes y servicios asociados en su hogar, como internet o dispositivos digitales, $y$, por ende, repercutir relativamente en el aprendizaje de sus hijos.

Una vez estimadas estas informaciones, se tomaron en cuenta aspectos relacionados con lo económico, lo social y lo educativo, así como laboral.

\subsection{Aspecto laboral}

Para medir esta dimensión, se tomaron en cuenta aquellos aspectos laborales formulados por el DANE (2019). Es por esto que se presenta el análisis correspondiente a la apreciación de los trabajadores de informalidad como la realidad laboral y el escenario laboral ante el Covid-19 (ver Tabla 1).

Tabla 1

Impacto laboral en contexto de informalidad en tiempos de pandemia

\begin{tabular}{|c|c|c|c|c|}
\hline & Ítem & Opciones & No & $\%$ \\
\hline \multirow{12}{*}{$\begin{array}{l}\bar{\pi} \\
\overline{0} \\
\frac{0}{0} \\
\frac{0}{0} \\
\frac{\pi}{0} \\
\frac{.0}{\pi} \\
\stackrel{0}{\simeq}\end{array}$} & \multirow{3}{*}{ 8. ¿usted ha sido despedido de su empleo? } & $\mathrm{Si}$ & 53 & $26,6 \%$ \\
\hline & & No & 63 & $31,7 \%$ \\
\hline & & No aplica / cuenta propia & 83 & $41,7 \%$ \\
\hline & \multirow{3}{*}{ 9.Fui obligado(a) a cerrar mi negocio } & $\mathrm{Si}$ & 55 & $27,6 \%$ \\
\hline & & No & 43 & $21,6 \%$ \\
\hline & & No aplica / cuenta propia & 101 & $50,8 \%$ \\
\hline & \multirow{3}{*}{$\begin{array}{l}\text { 10.¿Dejó de recibir pago por alquiler de } \\
\text { inmueble o vehículo? }\end{array}$} & $\mathrm{Si}$ & 39 & $19,6 \%$ \\
\hline & & No & 35 & $17,6 \%$ \\
\hline & & No aplica / cuenta propia & 125 & $62,8 \%$ \\
\hline & \multirow{3}{*}{ 11. ¿Usted laboralmente se encuentra? } & Ocupado & 61 & $30,7 \%$ \\
\hline & & Desocupado & 88 & $44,2 \%$ \\
\hline & & Cesante & 50 & $25,1 \%$ \\
\hline \multirow{5}{*}{ 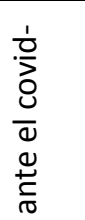 } & \multirow{5}{*}{$\begin{array}{l}\text { 12. ¿Cuál cree que sea la posibilidad de que } \\
\text { usted permanezca o regrese a su trabajo } \\
\text { habitual?" }\end{array}$} & Casi imposible & 60 & $30,2 \%$ \\
\hline & & Poco posible & 55 & $27,6 \%$ \\
\hline & & Algo posible & 56 & $28,1 \%$ \\
\hline & & Bastante posible & 8 & $4,0 \%$ \\
\hline & & Seguramente posible & 20 & $10,1 \%$ \\
\hline \multirow{6}{*}{ 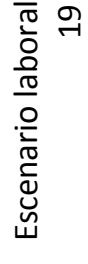 } & \multirow{6}{*}{$\begin{array}{l}\text { 13. ¿Usted está realizando algún cambio } \\
\text { significativo en su trabajo habitual? }\end{array}$} & Está haciendo teletrabajo & 21 & $10,6 \%$ \\
\hline & & Trabajando con normalidad & 12 & $6,0 \%$ \\
\hline & & Trabajando con turno $u$ horario & & \\
\hline & & especial & 21 & $10,6 \%$ \\
\hline & & Cuenta propia & 20 & $10,1 \%$ \\
\hline & & En casa sin trabajar & 125 & $62,8 \%$ \\
\hline
\end{tabular}

La tabla 1 muestra los ítems 8, 9, 10 y 11 relacionados con la realidad laboral de los ciudadanos que trabajan en contexto de informalidad. Así como los ítem 12 y 13, referentes a la situación laboral de dichos trabajadores ante el Covid-19. Se comprobó para el ítem 8 , que $26,6 \%$ de los encuestados si fueron despedidos de sus empleos, mientras que $31,7 \%$ indicaron que no han sido despedidos. El otro $41,7 \%$ dice que no aplica para ellos porque trabajan por cuenta propia. En lo concerniente al ítem 9, se confirmó que 27,6\% de los ciudadanos encuestados manifestaron que sí fueron obligados a cerrar sus negocios. Entretanto, 21,6\% dijo que no. El otro 50,8\% expuso que no aplica para ellos. Por otra parte, se evidenció en el ítem 10, que 19,6\% de los encuestados mencionaron 
que sí dejaron de recibir pago por alquiler de inmueble o de vehículo alquilado. Un 17,6\% dijo que no era su situación y otro $62,8 \%$ expresó que aplica para ellos. Los anteriores ítems inciden directamente sobre la opinión de la pregunta 11. De este modo, se conoció que en el ítem 11, los ciudadanos encuestados del contexto de informalidad se encuentran laboralmente ocupados (30,7\%); por otro lado, se hallan los desocupados $(44,2 \%)$ y los cesantes (25,1\%).

Estos resultados obenidos permiten deducir que la epidemia del Covid-19 junto a las crisis económicas pueden tener un impacto desproporcionado en los segmentos más vulnerables de la población, como es el contexto de informalidad, el cual es inestable laboralmente (Álvarez et al., 2020). De allí que se evidencie un alto margen de desocupados y cesantes por una contracción en la actividad económica de la región (CEPAL-OIT, 2020).

En cuanto al ítem 12, se encontró que $57,82 \%$ piensa que es casi imposible o poco probable que perdure o regrese a su trabajo habitual, mientras que $28,1 \%$ manifestó que tiene una alguna posibilidad de que se mantenga o retorne a sus labores habituales. El resto, $14,1 \%$ dice que su estabilidad o reintegro a su trabajo habitual es bastante o seguramente posible.

En lo relacionado con el ítem 13, se comprobó que los trabajadores de informalidad en la Ciudad de Cúcuta están efectuando cambios en su trabajo habitual característicos, ante el escenarios laborales en tiempo de Covid-19, pues 10,6\% está haciendo teletrabajo, mientras que 10,6\% está trabajando con horario especial. Otro 6\% está trabajando con normalidad y $10,1 \%$ lo está haciendo por cuenta propia; aunque, un $62,8 \%$ está en casa sin trabajar.

Los hallazgos de los ítems 12 y 13 ratifican que la realidad laboral y escenario ante Covid-19, puede provocar un empeoramiento de la desigualdad en el contexto de informalidad. Donde se acentúa el desempleo porque hay una disminición de la población ocupada de este contexto, lo cual concuerda con los datos suministrados por el DANE (2020), y coincide con la CEPAL (2020), quien señala que el Covid-19, perturba la tasa de ocupados y empleos, así como la calidad del trabajo, con lo cual podría haber una reducción en los salarios en los grupos más vulnerables, como son los trabajadores en el sector informal, y ver la poca posibilidad de permanecer en sus puestos de trabajo. Esto significa la desarticulación en el escenario laboral entre el desempleo y la informalidad (García, 2008). En consecuencia, este sector laboral afronta devastadoras consecuencias sociales y económicas dentro de la crisis sanitaria; incluidas las educativas.

\subsection{Aspecto económico}

Dentro de los aspectos formulados, se consideraron la realidad y situación económica del hogar y personal. Por ello, se presenta el análisis correspondiente a la apreciación de los ciudadanos del contexto de informalidad (Tabla 2). 
Tabla 2

Impacto económico en contexto de informalidad en tiempos de pandemia

\begin{tabular}{|c|c|c|c|c|}
\hline & Ítem & Opciones & No & Porcentaje \\
\hline \multirow{10}{*}{ 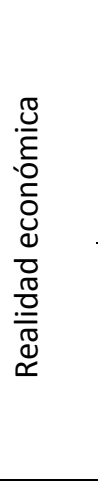 } & \multirow{5}{*}{$\begin{array}{l}\text { 14. ¿Cuál ha sido el grado de satisfacción con los } \\
\text { ingresos obtenidos en su hogar durante la } \\
\text { pandemia? }\end{array}$} & Totalmente satisfecho & 24 & $12,1 \%$ \\
\hline & & Muy satisfecho & 16 & $8,0 \%$ \\
\hline & & Satisfecho & 21 & $10,6 \%$ \\
\hline & & Un poco satisfecho & 61 & $30,7 \%$ \\
\hline & & Nada satisfecho & 77 & $38,7 \%$ \\
\hline & \multirow{5}{*}{$\begin{array}{l}\text { 15. ¿Cómo es la realidad económica del hogar } \\
\text { durante la pandemia? }\end{array}$} & Muy buena & 2 & $1,0 \%$ \\
\hline & & Buena & 10 & $5,0 \%$ \\
\hline & & Ni buena ni deficiente & 37 & $18,6 \%$ \\
\hline & & Deficiente & 76 & $38,2 \%$ \\
\hline & & Muy deficiente & 74 & $37,2 \%$ \\
\hline \multirow{8}{*}{ 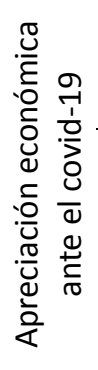 } & \multirow{3}{*}{$\begin{array}{l}\text { 16. ¿cómo percibe que será el futuro próximo en } \\
\text { cuanto a la situación económica del hogar? }\end{array}$} & Será mejor & 20 & $10,1 \%$ \\
\hline & & Seguirá igual & 127 & $63,8 \%$ \\
\hline & & Será peor & 52 & $26,1 \%$ \\
\hline & \multirow{5}{*}{$\begin{array}{l}\text { 17. ¿Cómo se verá afectada su situación económica } \\
\text { personal, en la pandemia Covid-19? }\end{array}$} & Muy afectada & 68 & $34,2 \%$ \\
\hline & & Bastante afectada & 71 & $35,7 \%$ \\
\hline & & Algo afectado & 50 & $25,1 \%$ \\
\hline & & Poco afectada & 9 & $4,5 \%$ \\
\hline & & Nada afectada & 1 & $0,5 \%$ \\
\hline
\end{tabular}

La tabla 2 muestra los ítems del 14 al 17, los cuales hacen referencia a la realidad económica y a la apreciación económica en el contexto de informalidad en tiempos de Covid-19; se reveló para el ítem 14, que entre las alternativas un poco satisfecho y nada satisfecho, 69,4\% de los encuestados manifestan una insatisfacción con los ingresos obtenidos de su trabajo y que redunda en beneficios para su hogar; mientrasel resto en relación a los ingresos de salarios se encuentran satisfechos (10,6\%); muy satisfechos (8\%), y para otros se encuentran totalmente sastifechos $(12,1 \%)$ con lo sueldos obtenidos. Lo cual está en concordancia con la hallado en el caso del ítem 15 , donde se detectó que $75,4 \%$ de los trabajadores de informalidad declararon que la realidad económica del hogar se encuentra entre deficiente y muy deficiente durante la pandemia Covid-19.

En tanto que $18,6 \%$ indicó que la situación económica del hogar ni es buena ni es deficiente. El resto, $6 \%$, halló que la situación del hogar está económicamente buena y muy buena.

Al determinar los resultados, en los ítems 14 y 15, se infiere que los trabajadores que se encuentran entre las poblaciones más frágiles frente a los efectos de la pandemia, está teniendo en los sectores informales de repercusiones negativas asociada a insatisfacción con bajos ingresos y, por lo tanto, con una baja capacidad de ahorro (Álvarez et al., 2020).

En relación al ítem 16, reveló que la apreciación de los encuestados en cuanto a la situación económica del hogar seguirá igual en el futuro próximo (63,8\%). Otros trabajadores de informalidad aprecian que será peor $(26,1 \%)$ y, el resto, considera que será mejor (10,1\%). Esta apreciación se mantiene en los resultados del ítem 17, donde se comprobó que la situación económica personal de los encuestados se muestra algo afectada, bastante afectada y muy afectada en un $95 \%$, mientras que el otro grupo de encuestados (5\%) percibe la situación económica personal poca afectada y nada afectada.

Al considerar los ítems 16 y 17, se infiere que los trabajadores de contexto de informalidad siguen estando originalmente vulnerables a las crisis económicas y laborales de la pandemia, puesto que, muchos de estos trabajadores han perdido sus empleos y enfrentan una situación de pobreza e inseguridad en la medida en que se intentifican los controles para mitigar el Covid-19, lo cual afecta a los trabajadores de informalidad. Estos 
individuos están subyugados a una relación imprevisible e irregular entre patrones y trabajadores. En el peor de los casos, el contexto de informalidad podría enfrentar un incremento negativo para el país y la ciudad de Cúcut, por la tasa de informalidad en el departamento y Cúcuta (Cámara de Comercio de Cúcuta, 2019a ). Lo que pudiera causar restricciones y limitaciones en el acceso a alimentos, bienes y servicios esenciales como acceso confiable a una educación desde la casa con uso de tecnología, debido al cierre de escuelas.

\subsection{Aspecto educativo}

Se muestra el análisis correspondiente a las percepciones de los participantes en torno al impacto del Covid-19 en el ámbito socioducativo (Tabla 3).

Tabla 3

Apreciaciones sobre impactos del Covid-19 en el ámbito socioeducativo, en contexto de informalidad

\begin{tabular}{|c|c|c|c|c|}
\hline & Ítem & Opciones & No & Porcentaje \\
\hline \multirow{11}{*}{ 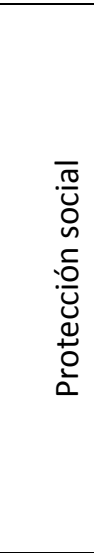 } & \multirow{2}{*}{$\begin{array}{l}\text { 18. ¿Actualmente en su hogar, } \\
\text { hay niños, niñas y adolescente } \\
\text { que se encuentran estudiando? }\end{array}$} & Si & 127 & $63,8 \%$ \\
\hline & & No & 72 & $36,2 \%$ \\
\hline & \multirow{5}{*}{$\begin{array}{l}19 \text { respecto al programa PAE, } \\
\text { ¿Su representado ha sido } \\
\text { beneficiado con el programa } \\
\text { durante la cuarentena? }\end{array}$} & Muy frecuentemente & 3 & $1,5 \%$ \\
\hline & & Frecuentemente & 160 & $80,4 \%$ \\
\hline & & Ocasionalmente & 0 & $0 \%$ \\
\hline & & Raramente & 0 & $0 \%$ \\
\hline & & Nunca & 36 & $18,1 \%$ \\
\hline & \multirow{4}{*}{$\begin{array}{l}\text { 20. ¿Usted o algún miembro de } \\
\text { su hogar recibe beneficios por } \\
\text { parte del Estado colombiano? }\end{array}$} & Familia en acción & 36 & $18,1 \%$ \\
\hline & & Colombia Mayor & 4 & $2,0 \%$ \\
\hline & & No aplica & 129 & $64,8 \%$ \\
\hline & & Jóvenes en acción & 30 & $15,1 \%$ \\
\hline \multirow{10}{*}{ 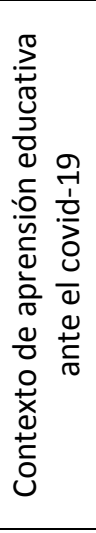 } & \multirow{6}{*}{$\begin{array}{l}\text { 21. ¿Usted o algún miembro de } \\
\text { su hogar ha tenido que recurrir a } \\
\text { familiares o amigos para } \\
\text { sastifacer las actividades } \\
\text { educativas remotas o a distancia } \\
\text { de sus representados en la } \\
\text { pandemia? }\end{array}$} & Muy frecuentemente & 32 & $16,1 \%$ \\
\hline & & Frecuentemente & 37 & $18,6 \%$ \\
\hline & & Ocasionalmente & 57 & $28,6 \%$ \\
\hline & & Raramente & 42 & $21,1 \%$ \\
\hline & & Nunca & 31 & $15,6 \%$ \\
\hline & & Me preocupan ambas opciones & 157 & $78,9 \%$ \\
\hline & \multirow{4}{*}{$\begin{array}{l}\text { 22. ¿Qué le preocupa más, el } \\
\text { coronavirus o la situación } \\
\text { educativa de sus } \\
\text { hijos/sobrinos/nietos? }\end{array}$} & Me preocupa poco ambas opciones & 1 & $0,5 \%$ \\
\hline & & No me preocupa ni el coronavirus ni la educativa & 5 & $2,5 \%$ \\
\hline & & Me preocupa más el coronavirus & 5 & $2,5 \%$ \\
\hline & & Me preocupa más mi situación educativa & 31 & $15,6 \%$ \\
\hline \multirow{10}{*}{$\begin{array}{l}\frac{1}{0} \\
\frac{0}{0} \\
\frac{\pi}{0} \\
\frac{0}{0}\end{array}$} & \multirow{5}{*}{$\begin{array}{l}\text { 23. Desde su punto de vista, } \\
\text { ¿Cuál es la realidad con sus } \\
\text { hijos/sobrinos/nietos aprende } \\
\text { desde casa en tiempos de } \\
\text { pandemia? }\end{array}$} & Adecuada y sin problemas & 29 & $14,6 \%$ \\
\hline & & Con algunos problemas & 100 & $50,3 \%$ \\
\hline & & Es una situación más dura de lo normal & 31 & $15,6 \%$ \\
\hline & & Está siendo muy duro & 19 & $9,6 \%$ \\
\hline & & Con deficiencias y muchos problemas & 20 & $10,1 \%$ \\
\hline & \multirow{5}{*}{$\begin{array}{l}\text { 24. ¿Cree que la educación } \\
\text { desde casa es efectiva para el } \\
\text { avance académico de sus } \\
\text { hijos/sobrinos/nietos? }\end{array}$} & Muy efectiva & 7 & $3,5 \%$ \\
\hline & & Efectiva & 24 & $12,1 \%$ \\
\hline & & Moderadamente efectiva & 60 & $30,2 \%$ \\
\hline & & Poco efectiva & 72 & $36,2 \%$ \\
\hline & & Efectivamente deficiente & 36 & $18,1 \%$ \\
\hline
\end{tabular}

A continuación se muestran los resultados de la tabla 4: Se logró identificar que para el ítem 18,63,8\% de los encuestados manifiesta que en sus hogares viven niños, niñas o adolecesntes que se encuentran estudiando. 
Asimismo, se determinó en el ítem 19, que 80,4\% expresó que con frecuencia su representado es beneficiado por el Programa Escolar de Alimentación (PAE), mientras que 18,1\% declaró que nunca. Lo cual pudiera deberse a que en el ítem 18 , un $36,2 \%$ declaró que en sus casas no hay niños, niñas o adolecesntes estudiando. En cuanto al ítem 20, se confirmó que algunos miembros del hogar de los encuestados reciben beneficios por parte del Estado colombiano, y se benefician de Familia en Acción (18,1\%); Colombia Mayor (2,0\%) y Jóvenes en Acción $(15,1 \%)$. El resto manifestó no recibir ningún beneficio o no aplica $(64,8 \%)$.

De este modo, se evidencia en los resultados hallados el apoyo brindado por el Gobierno de Colombia a través de programas de Protección social al otorgar beneficios a los estudiantes, aunque se observa que un grupo no es beneficiado. Lo cual concuerda con García \& Kairuz (2020) quienes señalan que los estudiantes matriculados en los colegios oficiales son beneficiarios del Programa de Alimentación Escolar (PAE) y el programa Familias en Acción ; aunque es posible que en la focalización de estas medidas se deje por fuera una parte importante de la población vulnerable. Además, en el sistema educativo oficial, de acuerdo con las medidas adoptadas, los estudiantes deberán ser atendidos a través de las 96 Secretarías de Educación certificadas (Decreto 470, 2020).

En relación al ítem 21, se pudo conocer que entre las alternativas muy frecuentemente, frecuentemente, 34,7\% de los encuestados manifestó que ha tenido que recurrir a familiares o amigos para sastifacer las actividades educativas remotas o a distancia de sus representados en la pandemia. Otro $28,6 \%$ dijo que lo hizo ocasionalmente. El resto, reveló que recurrió a amigos o a familiares, debido a actividades educativas de sus hijos/sobrino o nietos: raramente $(21,1 \%)$ y nunca( $15,6 \%)$.

No obstante, en el ítem 22, estos encuestados manifestaron que les preocupa el coronavirus y la situación educativa de sus hijos/sobrinos/nietos en 78,9\% de los casos. Entretanto, para el ítem 23, se demostró que los encuestados están presentando algunos problemas (50,3\%) sobre la realidad que tienen con el aprendizaje desde casa de sus hijos/sobrinos/nietos, con la educación desde el hogar en tiempos de pandemia y las actividades estudiantiles de estos. Otros encuestados manifestaron que es una situación más difícil de lo normal aprender desde casa (15,6\%); el resto dijo que está siendo muy difícil la situación $(9,6 \%)$ de la casa una escuela. Otro $(10,1 \%)$ dice que con deficiencias y muchos problemas, y solo $14,6 \%$ menciona que es adecuada y sin problemas.

No obstante, en el ítem 24, se determinó que la percepción de efectividad que tiene la educación desde casa para el avance académico de sus hijos/sobrinos/nietos es poco efectiva (36,2\%), y efectivamente deficiente $(18,1 \%)$. El resto tiene una apreciación más positiva sobre los estudios desde la casa con apoyo de los padres y familiares, siendo muy efectiva (3,5\%); otros dicen que será efectiva en $12,1 \%$ y moderadamente efectiva, 30,2\%.

Atendiendo los ítems 21, 22, 23 y 24, se deduce que en el contexto de informalidad, la crisis puede potencialmente exacerbar los agravios, la desconfianza y la sensación de injusticia sobre el acceso a los servicios de educación con tecnología a distancia o remota, porque se presenta un contexto de aprensión ante el Covid19 y brechas educativas (Murillo \& Duk, 2020), lo cual puede suscitar resultados negativos y perjudiciales para los estudiantes por el desgaste que tienen los padres y los docentes (Britez, 2020), o en la enseñanza desde la casa sin la preparación pedagógica de los padres, tíos, abuelos o hermanos puede ser débiles e inconsistentes (Razeto, 2016); así como también por la falta de estrategias que apoyen a los estudiantes de manera efectiva y promuevan aprendizajes significativos, o por la tenencia y uso de TIC en hogares que según el DANE (2018) la proporción porcentual de hogares a nivel nacional que posee computador de escritorio es de $28,8 \%$, portátil $(20,6 \%)$ o tableta $(10,9 \%)$ y conexión a Internet a nivel nacional es Hogares con Internet $(52,7 \%)$; Hogares con Internet - Fijo (40,5\%) y Hogares con Internet - Móvil (29,2\%). De allí las apreciaciones observadas en cuando a la educación desde casa y los problemas que acusan los trabajadores en contexto de informalidad. 


\section{Conclusiones}

Al reflexionar sobre la distribución de la informalidad, diferenciando entre ellos los ocupados, desocupados y cesantes, se encontró que la incidencia de la informalidad es mayor que las reflejadas en los resultados encontrados y en aquellos a los que se cuentan como más vulnerables en la fuerza laboral. La crisis de la pandemia, el aislamiento social obligatorio y la emergencia educativa interactúan con la complejidad existente en la tenencia y capacidad de generación de ingresos en los trabajadores, las circunstancias laborales, el acceso a bienes y servicios, acceso a educación de calidad, entre otros, haciendo que los individuos que forman parte del contexto de informalidad sean particularmente vulnerables a una paralización económica con respuestas poco convencionales.

En este sentido, dos dimensiones de fragilidad económica se unen para este contexto que impactan directamente el ámbito socioeducativo de los estudiantes. Primero, una mayor incidencia de informalidad que genera grandes riesgos de infección debido a la naturaleza de sus ocupaciones, por lo que son más sensibles a un menor grado en sus actividades de apoyar a los estudiantes en su deberes escolares desde casa debido al bloqueo y cierre de escuelas. Segundo, un grupo significativamente representativo de aproximadamente $57 \%$ que no supera una educación secundaria, cuyas consecuencias son particularmente graves para los estudiantes desfavorecidos y sus familias, porque el cierre de escuelas priva a los niños y jóvenes de oportunidades de desarrollo cognitivo y nutricional.

De este modo, los bajos ingresos económicos del contexto de informalidad, muchos niños, niñas y adolescentes dependen de programas escolares gratuitos para alimentarse de manera saludable. Por lo tanto, el cierre de las escuelas tiene un impacto en la nutrición escolar beneficiaria del Programa de Alimentación Escolar (PAE).

En cuanto a los padres que faciliten el aprendizaje en el hogar, se encontró en las percepciones de los trabajadores de informalidad que presentan dificultades para hacer frente a esta tarea. En este particular padres con poca educación y recursos limitados para enseñar desde casa. Además de acceso insuficiente a las tecnologías para la continuidad del aprendizaje, por lo cual, el contexto puede influir en que los niños, niñas y jóvenes no regresen a la escuela luego de la pandemia por el tipo de ocupación laboral de sus padres y familiares , lo que podría incidir en deserción escolar y aumento de trabajo infantil.

Esta dinámica es particularmente preocupante en el contexto socioeducativo con una alta incidencia de empleo, desempleo en la informalidad y la inestabilidad de la ocupación formal que tienen implicaciones económicas, sociales y educativas con cierre de escuelas, un hogar una escuela y el aprender desde casa, a su vez pérdidas de ingresos y accesos a medios digitales para reducir los efectos del distanciamiento social en el aspecto educativo porque los hogares colombianos muchas veces no cuentan con dispositivos electrónicos como computadora e internet, con lo cual se dificulta la educación a distancia y remota implementada a causa de la emergencia educativa generada por el Covid-19.

De allí se concluye que las desigualdades sociales, económicas y educativas en este contexto crean barreras y limitaciones para la implementación exitosa de educación a distancia en medio de emergencias. Aunado a las complicaciones de adapatación tanto de los padres, docentes y estudiantes de esta modalidad de estudio. Por ello, se involucran nuevas aristas a los problemas académicos existentes de bajo rendimiento, bajos logros y falta de interés por las asignaturas, que incluyen tanto al profesor como al estudiante y afectan el aprendizaje y la enseñanza, en medio de una crisis socioeconómica educativa del contexto de informalidad.

\section{Bibliographic references}

Álvarez, A., León, D, Medellín, M, Zambrano, A. \& Zuleta, H. (2020). Coronavirus in Colombia: vulnerability and policy options. Universidad de los Andes, Bogota, Colombia. 
https://www.undp.org/content/dam/rblac/Policy\%20Papers\%20COVID\%2019/undp-rblac-CD19-PDSNumber11-ES-Colombia.pdf

Arias, F. (2012).El Proyecto de Investigación Introducción a la metodología científica. Caracas, Venezuela: Editorial Episteme.

Bernal, C. (2010) Metodología de la Investigación. Para administración, economía, humanidades y ciencias sociales. México: Pearson.

Britez, M. (2020). La educación ante el avance del COVID-19 en Paraguay.Comparativo con países de la Triple Frontera. SciELO - Scientific Electronic Library Online. https://doi.org/10.1590/SciELOPreprints.22

Cámara de Comercio de Cúcuta (2019a). Impacto económico de la migración sobre la economía de Norte de Santander. http://www.cccucuta.org.co/media/reportecierrefrontera.pdf

Cámara de Comercio de Cúcuta (2019b). Informe de estudio económico de las zonas vigencia diciembre 2019. http://www.cccucuta.org.co/media/Publicaciones/informe_estudio_economico_de_la_zona_dic_2019.pd f

Comisión Económica para América Latina y el Caribe (CEPAL). (2020). Informe Especial COVID-19, No. 1. América Latina y el Caribe ante la pandemia del COVID-19 Efectos económicos y sociales. Naciones Unidas. https://repositorio.cepal.org/bitstream/handle/11362/45337/4/S2000264_es.pdf

Comisión Económica para América Latina y el Caribe (CEPAL) - Organización Internacional del Trabajo (OIT). (2020).El trabajo en tiempos de pandemia: desafíos frente a la enfermedad por coronavirus (COVID19).(Coyuntura Laboral en América Latina y el Caribe, $n^{\circ}$. 22). Naciones Unidas/OIT. https://repositorio.cepal.org/bitstream/handle/11362/45557/4/S2000307_es.pdf

Departamento Administrativo Nacional de Estadística (DANE). (2019). Boletín Técnico. Indicadores básicos de tenencia y uso de Tecnologías de la Información y Comunicación - TIC 2018 en hogares y personas de 5 y más años de edad.

https://www.dane.gov.co/files/investigaciones/boletines/tic/bol_tic_hogares_departamental_2018.pdf

Departamento Administrativo Nacional de Estadística (DANE). (2020). Boletín Técnico. Medición de empleo informal y seguridad social. Mayo 2020.

https://www.dane.gov.co/files/investigaciones/boletines/ech/ech_informalidad/bol_ech_informalidad_m ay20.pdf

Gambau, V. (2020). COVID-19: La crisis ha afectado a todos. Revista Española de Educación Física y Deportes, 429, 15-18. https://www.reefd.es/index.php/reefd/article/view/896/742

García, G. (2008). Informalidad regional en Colombia: Evidencia y determinantes. Desarrollo y Sociedad, (61), 43-85. https://doi.org/10.13043/dys.61.2

García, J. \& Kairuz, J. (2020). COVID-19 acciones globales frente al cierre de escuelas durante una pandemia.Bogota: Universidad de los Andes.

https://uniandes.edu.co/sites/default/files/asset/document/covid-19-acciones_globales-cierreescuelas.pdf

Hernández, R., Fernández, C. \& Baptista, M. (2014). Metodología de la Investigación. México: McGraw-Hill.

Ministerio de Hacienda y Crédito Público. (2020, 8 de mayo). Decreto Legislativo 639 de 2020. Por el cual se crea el Programa de apoyo al empleo formal - PAEF, en el marco del Estado de Emergencia Económica, Social y Ecológica declarado por el Decreto 637 de 2020.

https://dapre.presidencia.gov.co/normativa/normativa/DECRETO\%20639\%20DEL\%208\%20DE\%20MAYO\% 20DE\%202020.pdf 
Ministerio del Trabajo. (2020, 17 de marzo). Circular 021 de 2020. Medidas de protección al empleo con ocasión de la fase de contención de COVID-19 y dela declaración de emergencia sanitaria. https://www.mintrabajo.gov.co/documents/20147/0/Circular+0021.pdf/8049a852-e8b0-b5e7-05d3-

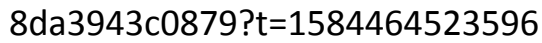

Ministerio del Trabajo. (2020, 27 de marzo). Decreto Legislativo 488 de 2020. Por el cual se dictan medidas de orden laboral, dentro del Estado de Emergencia Económica, Social y Ecológica. https://dapre.presidencia.gov.co/normativa/normativa/DECRETO\%20488\%20DEL\%2027\%20DE\%20MARZ O\%20DE\%202020.pdf

Ministerio de Trabajo. (2020, 04 de junio). Decreto 801 de 2020. Por medio del cual se crea el auxilio económico a la población cesante, en el marco del Estado de Emergencia Económica, Social y Ecológica. https://dapre.presidencia.gov.co/normativa/normativa/DECRETO\%20801\%20DEL\%204\%20DE\%20JUNIO\% 20DE\%202020.pdf

Montañez, C. E. (2016).Economía de la informalidad. Solución al problema del desempleo o causante de crisis económica. Revista Apuntes de Administración, 1(1), (60-67). https://revistas.ufps.edu.co/index.php/apadmin/article/view/995

Murillo, F. \& Duk, C.. (2020). El Covid-19 y las Brechas Educativas. Revista latinoamericana de educación inclusiva, 14(1), 11-13. https://dx.doi.org/10.4067/S0718-73782020000100011

Organización Internacional del Trabajo (OIT) (2020). Covid-19 y el mundo del trabajo: repercusiones y respuestas. https://www.ilo.org/wcmsp5/groups/public/---dgreports/--dcomm/documents/briefingnote/wcms_739158.pdf

Peralta, N., \& Bayona, J. P. (2016).La informalidad empresarial como barrera del crecimiento socioeconómico en Cúcuta. Revista Apuntes de Administración, 1(1), (29-36). https://revistas.ufps.edu.co/index.php/apadmin/article/view/990

Presidencia de la República de Colombia. (2020, 24 de marzo). Decreto 470 de 2020. Por el cual se dictan medidas que brindan herramientas a las entidades territoriales para garantizar la ejecución del Programa de Alimentación Escolar y la prestación del servicio público de educación preescolar, básica y media, dentro del Estado de Emergencia Económica, Social y Ecológica. Diario Oficial n. 51267. http://www.suinjuriscol.gov.co/viewDocument.asp?ruta=Decretos/30039002

Presidencia de la República. (2020, 17 de marzo). Decreto 417 de 2020. Por el cual se declara un Estado de Emergencia Económica, Social y Ecológica en todo el territorio Nacional.

https://dapre.presidencia.gov.co/normativa/normativa/DECRETO\%20417\%20DEL\%2017\%20DE\%20MARZ O\%20DE\%202020.pdf

Razeto, A. (2016). El involucramiento de las familias en la educación de los niños. cuatro reflexiones para fortalecer la relación entre familias y escuelas. Páginas de Educación, 9(2), 190-216.

https://doi.org/10.22235/pe.v9i2.1298

Sistema Nacional de Información de la Educación Superior (SNIES) (2018). Estadísticas generales de la Subdirección de Desarrollo Sectorial de Educación Superior. https://www.mineducacion.gov.co/sistemasdeinformacion/1735/w3-article-212352.html?_noredirect=1

Esta obra está bajo una Licencia Creative Commons Attribución-NoCommercial 4.0 International

(c) $)$ BY-NC 